\title{
Sensibilidad hepática a la insulina en residentes en zonas de altura y de nivel del mar utilizando el método CLAMPEH
}

\author{
Hepatic sensitivity to the insulin in residents from high-altitude and \\ sea level areas using the CLAMPEH method
}

Luis A. Aguilar Mendoza , Luis M. Baquerizo Sedano , Marilia L. Baquerizo Sedano

Universidad Continental

\section{RESUMEN}

Objetivos: Determinar y comparar la sensibilidad hepática a la insulina en residentes permanentes a nivel del mar (Lima, 150 msnm, Patm [presión atmosférica] $=760 \mathrm{~mm} \mathrm{Hg}$ ) y en altura (Huancayo, $3250 \mathrm{msnm}$, Patm $=520 \mathrm{~mm} \mathrm{Hg}$ ). Métodos: Se utilizó el método CLAMPEH, considerado el de mayor exactitud para determinar la sensibilidad a la insulina, y que consiste en la infusión por vía venosa, instalando dos catéteres antebraquiales de una cantidad fija de insulina y una cantidad variable de graduada con una bomba de infusión volumétrica. La sensibilidad hepática a la insulina se representó mediante el valor $(M)$, que indica la relación entre la cantidad de glucosa infundida con una hiperinsulemia para mantener valores de glicemia de $100 \mathrm{mg} / \mathrm{dL}$, siendo el valor estándar para esta relación 1 (en el grupo de edad de menos de 25 años y con 90-110\% del peso ideal). La muestra estuvo compuesta por 16 residentes permanentes por más de 5 años en cada ciudad, con un índice de masa corporal (IMC) entre 20 y 25 $\mathrm{kg} / \mathrm{m} 2$ y una edad entre 20 y 25 años. Resultados: Encontramos que la sensibilidad hepática a la insulina es significativamente mayor $(P<0,05)$ en el residente permanente de altura ( $\mathrm{m}$ valor $M=1,5231, \quad S D=0,3388$ ) respecto al residente permanente de nivel del mar ( $m$ valor $M=1,1092$, $\mathrm{SD}=0,2310$ ). Conclusiones: Planteamos que ello es consecuencia de una adaptación fisiológica y metabólica a la altura condicionada por la hipoxia y presión atmosférica.

1 Ph.D. Neurociencia y Biología del Comportamiento, director del Laboratorio de Neurociencia y Comportamiento de la Universidad Peruana Cayetano Heredia (UPCH). Investigador de la Universidad Continental.

2 Bachiller en Nutrición Humana, investigador asociado al Laboratorio de Neurociencia y Comportamiento, UPCH.

3 Bachiller en Psicología, investigadora asociada al Laboratorio de Neurociencia y Comportamiento, UPCH. 


\section{ABSTRACT}

Objectives: To determine and to compare the hepatic sensitivity to the insulin in permanent residents from sea level (Lima, 150 m.s.l, Patm [Atmospheric pressure] $=760 \mathrm{~mm} \mathrm{Hg}$ ) and from high-altitude (Huancayo, 3250 m.s.l, Patm $=520 \mathrm{~mm}$ $\mathrm{Hg})$. Methods: It was used the CLAMPEH method. It's considered the one with more accurately to determine the sensitivity to the insulin, which involves the infusion via venous, and to install two antebrachial catheters with an insulin fixed amount and a variable amount with a volumetric infusion pump. The hepatic sensitivity to the insulin was represented by the value $(M)$ which indicates the relationship between the glucose amount infused with a hyperinsulinemia, to maintain glycemia values of $100 \mathrm{mg} /$ $\mathrm{dL}$, being the standard value 1 for this relationship (in the age group under 25 and $90-110 \%$ of ideal weight). The sample was composed by 16 permanent residents for more than 5 years in each city, with a body mass index (BMI) between 20 and $25 \mathrm{~kg} / \mathrm{m} 2$ and an age between 20 and 25. Results: We found that the hepatic sensitivity to the insulin was significantly higher $(P<0.05)$ and the permanent resident from the highaltitude ( $\mathrm{m}$ value $M=1,5231, \mathrm{SD}=0.3388$ ) compared to the sea level permanent resident ( $m$ value $M=1,1092, S D=0,2310$ ). Conclusions: We propose that this is due to a physiological and metabolic adaptation at the conditioned height by the hypoxia and the atmospheric pressure.

Keywords: CLAMPEH, insulin, altitude, glycemia, hepatic sensitivity.

\section{INTRODUCCIÓN}

La resistencia a la insulina es una entidad clínica determinada por factores genéticos y ambientales, que se muestra con amplias variaciones, la sensibilidad a la insulina alterada es un factor de riesgo causante de diversas patologías y es un signo importante para identificar transtornos metabólicos, mantener niveles de glucosa en rangos de normalidad es el objetivo del tratamiento de la diabetes para evitar y/o retrasar los daños macrovasculares y microvasculares ocasionados por esta enfermedad, diversos estudios confirman que los residentes permanentes y nativos de la altura tienen una menor glicemia basal comparada con los habitantes del nivel del mar $(1,8)$. Se ha demostrado además que este fenómeno persiste durante los estados prandiales y permanece por los menos durante 12 horas de monitoreo continuo (9).

Aún se desconocen los mecanismos específicos que permiten explicar la menor glicemia en la altura (1). La regulación basal de la glicemia implica un proceso equilibrado de captación de glucosa y producción hepática de glucosa (PHG) (1). Como los sujetos de la altura y del nivel del mar tienen una similar insulinemia basal (10), el fenómeno podría ser atribuido a los mecanismos a nivel del receptor o posreceptor de insulina (9). Algunos estudios in vitro indican que la hipoxia mejora la captación de glucosa debido a que incrementa la translocación de los transportadores de glucosa (principalmente GLUT-4) en la célula muscular esquelética y cardiaca $(11,15)$.

Por otro lado, la regulación de la PHG depende de la tasa de glucogenolisis y gluconeogénesis (16), y en esta regulación intervienen por lo menos tres componentes: la insulina, los ácidos grasos libres (que cumplen un rol fundamental) y el glucagón $(17,18)$. Se ha estimado que la tasa de gluconeogénesis hepática en sujetos de la altura es mayor que en sujetos del nivel del mar (19), pero el método de flujo esplácnico usado por los respectivos investigadores no provee información exacta de la tasa de gluconeogénesis $(1,20)$.

Otros estudios refieren un incremento del $100 \%$ en la producción hepática basal de glucosa respecto al nivel inicial (21); sin embargo, no es seguro si estos resultados se pueden extrapolar a los sujetos que residen permanentemente en la altura, 
considerando que los cambios tempranos de adaptación en el organismo en respuesta a los cambios de altitud pueden involucrar a hormonas de estrés $u$ otros mecanismos que podrían influir en la tasa de PHG (1).

Aún es incierto que la menor glicemia en la altura se deba a una menor PHG, ya que no se tiene información acerca de la sensibilidad hepática a la insulina en residentes permanentes de la altura (1). Entendiendo que ésta es el efecto de la insulina sobre los tejidos sensibles a esta hormona para promover la captación de glucosa y suprimir la salida de glucosa del hígado.

Se han realizado estudios que hicieron uso de la prueba de tolerancia a la glucosa oral (4) e intravenosa $(5,6)$ y es por el método del clamp de la glucosa $(18,19)$ que demostraron que la sensibilidad a la insulina en altura es más alta. Sin embargo, es necesario realizar un estudio detallado con un método más adecuado. Por ello, desarrollamos en esta investigación un estudio comparativo en ambientes naturales, orientado a estimar la sensibilidad hepática a la insulina (SHI) en residentes permanentes en la altura y a nivel del mar, mediante el método clamp euglicémico hiperinsulémico - CLAMPEH (1).

\section{MATERIAL Y MÉTODOS}

La investigación fue de naturaleza básica y descriptiva, de diseño no experimental. El método específico utilizado para estimar la SHI fue el clamp euglicémico hiperinsulémico - CLAMPEH, considerado el "estándar oro" de los métodos que cuantifican la sensibilidad a la insulina in vivo.

Consiste en la infusión por vía venosa, instalando dos catéteres antebraquiales (Abbocath $\left.n^{\circ} 20,22\right)$, de una cantidad fija de insulina (solución de insulina Humalog de acción rápida en suero fisiológico $100 \mathrm{U} / \mathrm{L}$, en función del área de superficie corporal) y una cantidad variable de glucosa (solución de dextrosa al $10 \%$, en función de la glicemia inicial y su valor cada 5 minutos, estimada con un glucómetro y bandas reactivas) graduada con una bomba de infusión volumétrica ZNB-XD, con el fin de mantener la glucemia del sujeto en un valor de euglucemia prefijado $(100 \mathrm{mg} / \mathrm{dL}+/-5$ $\mathrm{mg} / \mathrm{dL}$ ) (22).

Los datos fueron recolectados en una ficha en la que se registra la edad, peso, talla, cantidad de glucosa infundida en $\mathrm{mg}$, cantidad de insulina infundida en m.u., el valor de glicemia cada 5 minutos y el valor de equilibrio (valor $M$ ). El valor $M$ representa la SHI, y resulta de dividir la cantidad de glucosa e insulina infundida en los 30 minutos siguientes después de llegar al valor de euglicemia prefijado. Este valor se expresa en $\mathrm{mg} / \mathrm{kg} / \mathrm{min}$ o en forma de coeficiente, siendo el valor 1 el resultado del clamp promedio en el grupo de edad de menos de 25 años y con 90-110\% del peso ideal $(22,23)$.

La muestra incluyó a 50 personas, que cumplían con los criterios de inclusión: intervalo de edad de 20 a 25 años, residentes permanentes por más de 5 años en Huancayo (3 250 msnm, Patm $=520$ $\mathrm{mm} \mathrm{Hg}$ ) y Lima (150 msnm, Patm $=760$ $\mathrm{mm} \mathrm{Hg}$ ) con un IMC entre 20 y $25 \mathrm{~kg} / \mathrm{m} 2$. Se trabajaron con los valores de 32 personas que lograron el equilibrio glicémico durante el tiempo requerido para que los datos sean considerados válidos. El promedio de edad de los residentes permanentes en altura fue de 22,62 y en el nivel del mar de 22,94.

\section{RESULTADOS}

En la Tabla $\mathrm{N}^{\circ} 1$ se muestra los datos obtenidos, la cantidad de dextrosa infundida, la cantidad de insulina infundida y el valor $M$, que es la relación entre ambas referentes a la sensibilidad hepática a la insulina (expresada en el valor $M$ ) en residentes permanentes en zonas de altura y de nivel del mar. 
Tabla №1: Dextrosa e insulina infundida y valor $M$, en residentes permanentes en la altura y a nivel del mar

\begin{tabular}{|c|c|c|c|c|c|c|}
\hline & Dextrosa (mg) & $\begin{array}{c}\text { Altura } \\
\text { Insulina (m.u.) }\end{array}$ & Valor M & Dextrosa (mg) & $\begin{array}{l}\text { Nivel del mar } \\
\text { Insulina (m.u.) }\end{array}$ & Valor $M$ \\
\hline \multirow{8}{*}{ Varones } & 15,0 & 864,0833 & 1,7359 & 10,5 & 1152,9961 & 0,9107 \\
\hline & 12,0 & 1223,2129 & 0,9810 & 18,0 & 1008,5237 & 1,7848 \\
\hline & 12,0 & 1069,6167 & 1,1218 & 12,0 & 1007,3728 & 1,1912 \\
\hline & 18,0 & 985,6368 & 1,8262 & 10,5 & 1035,6640 & 1,0138 \\
\hline & 15,0 & 1079,0922 & 1,3900 & 13,5 & 1026,1579 & 1,3156 \\
\hline & 15,0 & 987,9271 & 1,5183 & 12,0 & 1078,2949 & 1,1129 \\
\hline & 18,0 & 1052,9957 & 1,7094 & 10,5 & 1153,2562 & 0,9105 \\
\hline & 16,5 & 1097,2693 & 1,5037 & 9,0 & 1051,1898 & 0,8562 \\
\hline \multirow{8}{*}{ Mujeres } & 10,5 & 916,9732 & 1,1450 & 12,0 & 902,4965 & 1,3296 \\
\hline & 18,0 & 847,1127 & 2,1248 & 10,5 & 874,6428 & 1,2005 \\
\hline & 18,0 & 848,0566 & 2,1225 & 9,0 & 909,2854 & 0,9898 \\
\hline & 10,5 & 951,7825 & 1,1031 & 10,5 & 979,7959 & 1,0716 \\
\hline & 13,5 & 868,9073 & 1,5536 & 10,5 & 914,9863 & 1,1475 \\
\hline & 12,0 & 860,2325 & 1,3949 & 9,0 & 952,4705 & 0,9449 \\
\hline & 13,5 & 909,2854 & 1,4846 & 9,0 & 985,9006 & 0,9129 \\
\hline & 15,0 & 906,4215 & 1,6548 & 10,5 & 995,9920 & 1,0542 \\
\hline
\end{tabular}

En la Figura $\mathrm{N}^{\circ} 1$ comparamos la sensibilidad hepática a la insulina en residentes permanentes en la altura y a nivel del mar, hallamos los promedios ( $\mathrm{m}$ valor M) en ambos grupos, siendo 1,5231 para el grupo de altura y 1,1092 para el grupo de nivel del mar, y determinamos que la diferencia es estadísticamente significativa.

La prueba estadística utilizada fué la $†$ de Student $(P<0,05)$, determinándose que los valores tienen una correlación negativa utilizando la $r$ de Pearson (estadístico $r=$ $-0,504$, valor $P=0,047)$.

\section{DISCUSIÓN}

Estudios controlados, que utilizaron diversos métodos, sugerían una mayor sensibilidad hepática a la insulina en residentes permanentes en la altura en comparación con residentes permanentes a nivel del mar. Los resultados de esta investigación lo confirman mediante la prueba del CLAMPEH en ambientes naturales.

La mayor sensibilidad hepática a la insulina responde a la adaptación fisiológica y metabólica a la altura. La hipoxia y presión

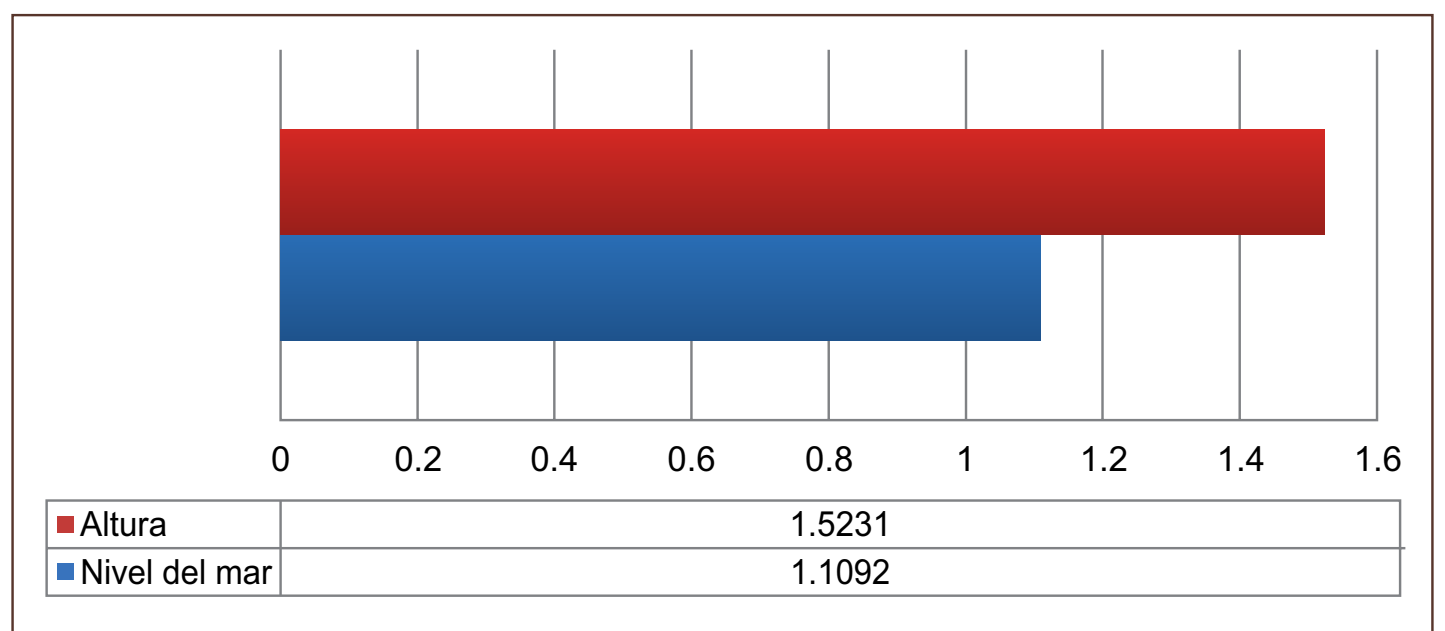

Figura $N^{\circ} 1$ : Comparación de promedio de valor $M$ en altura y a nivel del mar 
atmosférica en esta, condicionaría varios factores implicados específicamente en la regulación del metabolismo de glucosa. Para comprender mejor el sistema de regulación, especial atención ahora deben tener las alteraciones en el transporte de glucosa y los receptores de insulina.

La adaptación a condiciones de hipoxia y menor presión atmosférica se ve en nativos de la altura sin patologías asociadas, los cambios en la sensibilidad hepática a la insulina en nativos de nivel del mar en la etapa de acomodación y aclimatación a la altura aún se desconocen.

Los resultados corroboran el estudio previo realizado por Orison Woolcott y Oscar Castillo en 2008, en donde encontraron una tasa mayor de infusión de glucosa en los pobladores de las alturas(15,3 \pm 4,6 $\mathrm{mg} / \mathrm{kg} / \mathrm{min}$ ) que los habitantes del nivel del mar $(10,9 \pm 3,8 \mathrm{mg} / \mathrm{kg} / \mathrm{min})$ durante el clamp de glucosa realizado a $125 \mathrm{mg} / \mathrm{dL}$ por encima del valor basal obtenidos con el método clamp euglicémico hiperglicémico - CLAMPEH.

Los diversos cambios en la fisiología del nativo de altura están relacionados con la exposición crónica a la hipoxia, los cambios metabólicos causados por el HIF1 (Hypoxia-inducible factors) y la cascada de señalización que origina una alteración en los transportadores de glucosa GLUT-2 y GLUT-4 .

\section{REFERENCIAS BIBLIOGRÁFICAS}

1. Woolcott O, Castillo O. Metabolismo de la glucosa en el habitante de la altura: Replanteando evidencias. Arch Biol Andina. 2008; 14(1): 51-62.

2. San Martín M. Distribución de la glucosa sanguínea y su variación con el cambio de altitud. An Fac Med. 1940; 23(1): 312-318.

3. Monge CC. Glucosa, ácido láctico y ácido pirúvico a nivel del mar y en la altura. An Fac Med. 1949; 32(1): 6065.

4. Picón-Reátegui E. Studies on the metabolism of carbohydrates at sea level and at high altitudes. Metabolism. 1962; 11(11): 48-54.

5. Picón-Reátegui E. Intravenous glucose tolerance test at sea level and at high altitudes. J Clin Endocrinol Metab. 1963; 23(1): 1256-1261.

6. Calderón R, Llerena A. Carbohydrate metabolism in people living in chronic hypoxia. Diabetes. 1965; 14(1): 100105.

7. Garmendia F. Glicemia del nativo normal de altura. Arch Inst Biol Andina. 1970; 3(2): 09-16.

8. Heat D, Williams D. Man at high altitude: the pathophysiology of acclimatization and adaptation. $1^{\mathrm{a}}$ ed. New York: Churchill Livingstone: 1981.

9. Castillo O, Woolcott Oscar, Gonzáles V, Tello L, Villarreal C, et al. Monitoreo continuo de la glicemia en el poblador de los andes. Diagóstico. 2006; 45(1): 39-43.

10. Garmendia F, Torres J, Tamayo R, Urdanivia E. Carbohydrate metabolism at high altitude. Abstract book of the 8th Congress of the International Diabetes Federation. Brussels, Belgium. 1973; 262(1): 15-20.

11. Cartee G. Douen AG, Ramlal T, Klip A, Holloszy JO. Stimulation of glucose transport in skeletal muscle by hypoxia. J Appl Physiol. 1991; 70(4): 593-600.

12. Azevedo J, Carey J, Pories W, Morris P, Lynis G. Hypoxia stimulates glucose transport in insulinresistant human skeletal muscle. Diabetes. 1995; 44(1): 695-698.

13. Reynolds T, Brozinick J, Rogers MA, Cushman SW. Mechanism of hypoxiastimulated glucose transport in rat skeletal muscle: potential role of glycogen. Am J Physiol. 1998; 274: 773-778.

14. Zhang J, Behrooz A, Ismail-Beigi F. Regulation of glucose transport by hypoxia. Am J Kidney Dis. 1999; 34(1): 189-202.

15. Weickert $M$, Pfeiffer A. Signalling 
mechanisms linking hepatic glucose and lipid metabolism. Diabetologia. 2006; 49(8): 1732-41.

16. Bergman R. Non-esterified fatty acids and the liver: why is insulin secreted into the portal vein? Diabetologia. 2000; 43(7): 46-52.

17. Jiang $G$, Zhang B. Glucagon and regulation of glucose metabolism. Am J Physiol Endocrinol Metab. 1995; 284(4): 1-8.

18. Castillo O, Alzamora J, Capatinta C, et al. Insulin sensitivity at high altitude. Abstract book of the 5th European Congress of Endocrinology. 1998; 5(2): 9-13.

19. Torres J. Sensibilidad a la insulina en altura y a nivel del mar. High Alt Med Biol. 2002; 3(1): 97-138.

20. Marticorena E. Entidades nosológicas de desadaptación aguda a la altura. An Fac Med Lima. 1997; 58(2): 85-91.

21. Consensus Development Conference on Insulin Resistance. 5-6 November 1997. American Diabetes. Association. Diabetes Care. 1998; 21 (2): 310-314.

22. Pérez M, Montanya E. Técnicas para el estudio de la resistencia insulínica. Una valoración crítica. AV DIABETOL. 2001; 17(1): 179-186

23. Cabezas-Cerrato J, Araujo D. Resistencia a la acción de la insulina. Evolución histórica del concepto. Técnicas para el estudio in vivo en humanos; 2004. 